Cerebrovasc Dis 2009;28:205-206

DOI: $\underline{10.1159 / 000226582}$

\section{Alternative Ultrasound Windows and Sensitivity of Contrast Transcranial Doppler in Patients with Patent Foramen Ovale}

\section{Mehmet A. Topcuoglu}

Neurosonology Laboratory, Department of Neurology, Hacettepe University Hospitals, Ankara, Turkey

I read with great interest the article by Kobayashi et al. [1] regarding the accuracy of contrast transcranial Doppler (cTCD) in the diagnosis of right-to-left intracardiac shunts (RLS), mainly patent foramen ovale (PFO). First of all, I congratulate the researchers for their technical design for studying simultaneous CTCD and contrast transesophageal echocardiography (cTEE). However, I have some reservations about their method for CTCD examination, which should be mentioned because of significant effects on the interpretation of the results.

In this study, the authors analyzed 321 combined CTCD and cTEE examinations performed on 107 patients. Among 78 examinations with a small PFO detected by cTEE (less than 30 microbubbles, MBs, counted), cTCD detected MBs in only 19 (24\%). In contrast, of all 27 examinations with a large PFO found by cTEE, MBs were also found with cTCD. Based on ROC analysis, the details of which are not available to us, they declared that 2 or more MBs on CTCD is the cutoff for predicting a large PFO with cTEE. These results led them to state that CTCD can only diagnose large PFOs. Because this is written in the title of the article and in the conclusion, a causal reader could think that CTCD is not sensitive enough to detect small PFOs. This argument is not fully consistent with the related literature $[2,3]$, as discussed by the authors [1]. Potential confounders listed for this discrepancy by Kobayashi et al. [1] are differences in PFO size, number of contrast injections, contrast type, patients' positions and the minimum number of MBs required for the diagnosis. However, in my view, there is one more reason, which may perhaps be the most important one.

In the absence of an adequate temporal sonic bone window, they insonated the right internal cerebral artery (ICA) from the orbital window at a depth of 60-65 $\mathrm{mm}$. Interestingly, 45 of the 107 (42\%) patients underwent cTCD from temporal windows and the ICA was monitored in the remaining 62 (58\%). In other words, it can be said that temporal sonic bone window was inadequate in $58 \%$ of the patients studied. This extremely high prevalence of insufficient temporal window, in my view, represents a lower performance of CTCD that occurs only when it is performed simul- taneously with cTEE; this is because the experiment is designed for CTEE, not for CTCD. Therefore, the low diagnostic capability of cTCD for small PFOs may solely be due to this factor. Furthermore, transorbital monitoring of the carotid siphon was not sufficiently investigated. Therefore, this technique should be considered as an experimental procedure.

Because of mandatory manual fixation of the TCD probe during the Valsalva maneuver, it is quite possible to miss the Doppler signal during the most critical part of the test. As a neurosonologist who has performed these studies for many years, I can say that it is very difficult to reestablish a lost Doppler signal quickly in this test. Therefore, before declaring the superiority of cTEE in the detection of small PFOs, the authors should validate their method in appropriate settings. One can suggest that some safety problems may also be present with transorbital monitoring because of the possible adverse effects of vagal stimulation in this unwell population.

The bubbles test can be performed directly by insonation of the extracerebral arteries in cases with inadequate temporal bone windows for ultrasound. For this purpose, transorbital insonation of the carotid siphon was used in this study [1]. Likewise, cervical insonation of the common carotid artery [4] submandibular insonation of the internal carotid artery [5], transforaminal insonation of vertebral and basilar arteries [6-8] or even insonation of more peripheral arteries, such as the contralateral brachial or femoral arteries [9], can be performed. However, none of the studies tested the utility of these techniques in patients with insufficient transtemporal bone windows. Therefore, we cannot say that they are useful, although, in this specific indication, they could have been. In addition to cTEE, which is still considered as the gold standard for RLS detection, other echocardiographic methods such as transmitral Doppler [10] and insonation of the arcus aorta [11] were also proposed. However, these were also not tested in these subjects.

Among these alternative sites for sonographic MB monitoring, the submandibular (high cervical) ICA is perhaps the most suitable site. It has been shown that submandibular ICA recording is at least as sensitive and specific as middle cerebral artery monitoring with power M-mode Doppler TCD in the bubble test [5]. In this study [5], 77\% more MBs were detected in the submandibular ICA compared to the ipsilateral middle cerebral artery. The possible reason for this difference is that MBs decay before their arrival into intracranial arteries and entry into the anterior cerebral artery [12]. Thus, detection of more small RLS corresponds to higher diagnostic utility with submandibular ICA recording.

Again, it should be kept in mind that none of these alternative ways had been validated in the patients with insufficient temporal windows. Therefore, further studies are required before their entry into routine clinical practice.

\section{KARGER}

Fax +41613061234 E-Mail karger@karger.ch www.karger.com
(C) 2009 S. Karger AG, Base

$1015-9770 / 09 / 0282-0205 \$ 26.00 / 0$ 


\section{References}

1 Kobayashi K, Iguchi Y, Kimura K, Okada Y, Terasawa Y, Matsumoto N, Sakai K, Aoki J, Shibazaki K: Contrast transcranial Doppler can diagnose large patent foramen ovale. Cerebrovasc Dis 2009;27:230-234.

2 Di Tullio M, Sacco RL, Venketasubramanian N, Sherman D, Mohr JP, Homma S: Comparison of diagnostic techniques for the detection of a patent foramen ovale in stroke patients. Stroke 1993;24:1020-1024.

-3 Belvis R, Leta RG, Marti-Fabregas J, Cocho D, Carreras F, Pons-Llado G, Marti-Vilalta JL: Almost perfect concordance between simultaneous transcranial Doppler and transesophageal echocardiography in the quantification of right-to-left shunts. J Neuroimaging 2006;16:133138.

4 Draganski B, Blersch W, Holmer S, Koch H, May A, Bogdahn U, Hölscher T, Schlachetzki F: Detection of cardiac right-to-left shunts by contrast-enhanced harmonic carotid duplex sonography. J Ultrasound Med 2005;24:1071-1076.

5 Topcuoglu MA, Palacios IF, Buonanno FS: Contrast M-mode power Doppler ultrasound in the detection of right-to-left shunts: utility of submandibular internal carotid artery recording. J Neuroimaging 2003;13:315-323.

6 6 Perren F, Savva E, Landis T: Transforaminal Doppler: an alternative to transtemporal approach for right-to-left cardiac shunt assessment. J Neurol Sci 2008:273:49-50.

7 Del Sette M, Dinia L, Rizzi D, Sugo A, Albano B, Gandolfo C: Diagnosis of right-to-left shunt with transcranial Doppler and vertebrobasilar recording. Stroke 2007;38:2254-2256.
8 Morelli N, Galerini S, Cafforio G, Chiti A, Baldacci F, Mancuso M, Orlandi G, Tartaglione A, Murri L: Vertebrobasilar recording with contrast transcranial Doppler in right-to-left shunt diagnosis. Cerebrovasc Dis 2008; $26: 569$.

-9 Daly KJ, Hutchinson S, McCollum CN: Can a peripheral artery be used to detect venous-to-arterial circulation shunts? Cerebrovasc Dis 2007; 25:111-114

10 Fox ER, Picard MH, Chow CM, Levine RA, Schwamm L, Kerr AJ: Interatrial septal mobility predicts larger shunts across patent foramen ovales: an analysis with transmitral Doppler scanning. Am Heart J 2003; 145:730-736

11 Yasaka M, Ikeno K, Otsubo R, Oe H, Nagano K, Minematsu K: Rightto-left shunt evaluated at the aortic arch by contrast-enhanced transesophageal echocardiography. J Ultrasound Med 2005;24:155-159.

12 Topcuoglu MA: Diagnosis of right-to-left shunts by transcranial Doppler in patients with insufficient temporal bone window. Stroke 2008;39: e123-e124.

Mehmet A. Topcuoglu, MD

Neurosonology Laboratory, Department of Neurology

Hacettepe University Hospitals

TR-06100 Ankara (Turkey)

Tel. +90 312305 1806, Fax +90 3123093451

E-Mail matopcuoglu@yahoo.com 